\title{
A Low Cost CMOS Polarimetric Ophthalmoscope Scheme for Cerebral Malaria Diagnostics
}

\author{
Xiaojin Zhao ${ }^{\dagger, *}$, Amine Bermak ${ }^{\dagger}$, and Farid Boussaid ${ }^{\S}$ \\ ${ }^{\dagger}$ Dept. of ECE, The Hong Kong University of Science and Technology, Hong Kong \\ ${ }^{*}$ School of Electronic Sci. \& Tech., Shenzhen University, Shenzhen, P. R. China \\ ${ }^{\S}$ School of EECS, The University of Western Australia, Perth, Australia \\ eexjzhao@szu.edu.cn;eebermak@ust.hk;boussaid@ee.uwa.edu.au
}

\begin{abstract}
In this chapter, we present a low cost CMOS polarimetric ophthalmoscope scheme enabling the capture of the retinal abnormalities that are unique to cerebral malaria. The proposed technology, which can be integrated into cellphones, offers the basis for quick and non-invasive screening of cerebral malaria. In addition, we report a micropolarizer array technology exploiting "guest-host" interactions in liquid crystals for visible imaging polarimetry. The proposed technology enables the fabrication of high resolution $(5 \mu m \times 5 \mu m$ pixel pitch) micropolarizer arrays with submicron thickness $(0.95 \mu \mathrm{m})$ to provide for larger light collection angles and reduced optical cross-talk. With the "host" nematic liquid crystal molecules photo-aligned by sulfonic azo-dye SD1, we report averaged major principal transmittance and polarization efficiency (PE) of $80.3 \%$ and 0.863 , respectively across the $400 \mathrm{~nm}-700 \mathrm{~nm}$ visible spectrum range. The proposed fabrication technology is simple and costeffective, requiring only a single ultraviolet-exposure of the spin-coated "guest-host" mixture through a "photoalignment master".
\end{abstract}

Key words: Polarimetric ophthalmoscope; polarimetric imaging; micropolarizer array

\section{Introduction}

Cerebral malaria is an acute, widespread disease of the brain, which is accompanied by fever, cognitive impairments (especially in children) and coma. The mortality ratio is between $25-50 \%$. If a person is not treated, cerebral malaria is fatal in 24-72 hours. The direct visualization of the malaria parasite, under the microscope, remains the gold standard for its diagnosis. To remove the need for the required skilled and specialized equipment (microscope, reagents), rapid diagnostic tests have been proposed to detect the antigens/products of the parasite in the blood. However, these tests are often difficult to interpret because they exhibit lower sensitivities at low parasite densities and vary in sensitivity between malaria species [1]. To improve the diagnosis of cerebral malaria, we propose a low cost digital polarimetric ophthalmoscopy technology that enables 
the capture of the retinal abnormalities that are unique to cerebral malaria. The cost of ophthalmoscopes and the level of skills required (pupil dilatation and eye examination) do not allow currently for the wider use of ophthalmoscopy in poor malaria-endemic countries. To address these issues, we propose a low cost CMOS polarimetric ophthalmoscope scheme that enables a simple mobile phone to be used as a digital ophthalmoscope (Fig. 1). The user could ultimately even take a polarimetric snapshot of his/her own retina. The retinal images would then be transmitted to a processing node, which would then screen the data for signs of malarial retinopathy. Since the retina is part of the central nervous system, it exhibits a similar cellular structure and blood-tissue barrier. As a result, examining the retina provides a non-invasive and in vivo means to detect infected microvasculature and neurologic tissue [1]. It is widely accepted that cerebral malaria is manifested by retinal whitening and a discoloration of retinal vessels to orange or white [1]. Furthermore, other research works have shown that polarization imaging can provide an enhanced view of the tissue and reveal details not visible by conventional retinal photography [2].

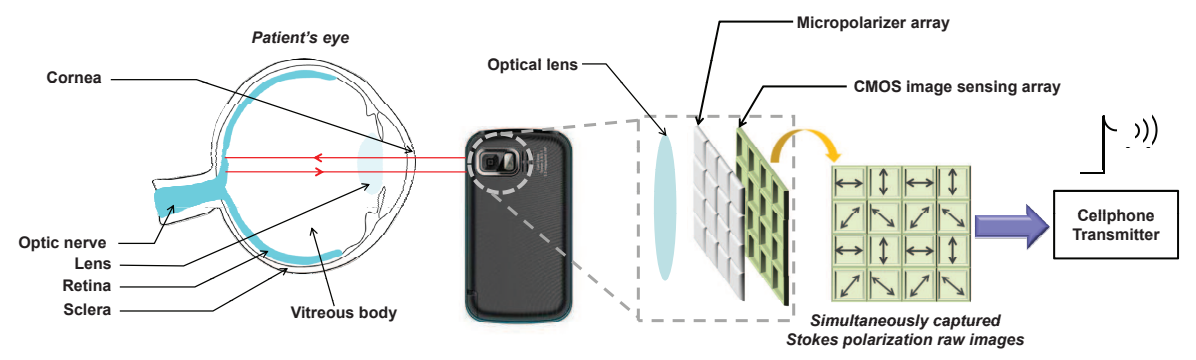

Fig. 1. Proposed low cost CMOS polarimetric ophthalmoscope scheme for cerebral malaria diagnostics.

As shown in Fig. 1, the integration of a micropolarizer array (i.e. a mosaic of micro-scale polarizer elements of different orientations) over the pixel array of an image sensor enables the concept of a low cost single-chip polarization camera capable of capturing, in a single frame, the polarimetric information of a scene [3]. A number of micropolarizer array (MPA) implementations have been demonstrated for image sensors. Examples include patterned dichroic films such as polyvinyl alcohol (PVA) $[4,5]$, birefringent $Y V O_{4}$ crystal covered by patterned aluminum films [6], multiple-domain liquid crystal with micro-patterned alignment layers [7], and nanometer-scale metal wire grid [8]. In each case, selective etching is used to pattern micropolarizer elements at the pixel pitch. To completely remove the need for this complex selective etching, we proposed, in [9], to use instead the well-controlled process of ultraviolet (UV) photolithography for the patterning of a high resolution pixel-level micropolarizer array. A spin-coated photosensitive azo-dye-1 (AD1) was used as the polarizing film [9]. This material exhibits a strong dichroism after sufficient exposure to linearly 
polarized UV light. The rod-like molecules of a spin-coated AD1 film can be oriented with their long molecular axes perpendicular to the orientation of the projected polarized UV light. However, the orientation degree of AD1 molecules is a function of the exposure energy and saturates after prolonged UV exposure. As a result, a UV-patterned polymer AD1 film exhibits limited major principal transmittance and extinction ratio [9].

In this chapter, a "guest-host" MPA, with photo-aligned polymerizable nematic liquid crystal (NLC) as the "host" material and AD1 molecules as the "guest", is proposed to improve the orientation degree of AD1 molecules and thus the optical performance. Section 2 presents the micropolarizer array pattern design. Section 3 describes the principle of a "guest-host" micropolarizer array and its fabrication. Experimental characterization results are discussed in Section 4. Finally, a conclusion is drawn in Section 5.

\section{Micropolarizer array pattern design}

In this section, we describe the pattern design methodology for the "guest-host" micropolarizer array. Light's partial linear polarization information is fully characterized by the first three Stokes parameters $\left(S_{0}, S_{1}, S_{2}\right)[10]$. With $\left(S_{0}, S_{1}, S_{2}\right)$ representing the total intensity, the amount of linear $0^{\circ}$ or $90^{\circ}$ polarization and the amount of linear $45^{\circ}$ or $-45^{\circ}$ polarization, respectively, the emerging light intensity after passing a linear polarizer can be expressed as a linear combination of $\left(S_{0}, S_{1}, S_{2}\right)$ with their coefficients as functions of the transmission axis orientation of the linear polarizer. This can be accurately described by introducing the linear polarizer's Mueller matrix $M_{\text {linear }}$ [10]:

$$
\left[\begin{array}{cccc}
\alpha & \beta \cos 2 \theta & \beta \sin 2 \theta & 0 \\
\beta \cos 2 \theta & \alpha \cos ^{2} 2 \theta+\gamma \sin ^{2} 2 \theta & (\alpha-\gamma) \sin 2 \theta \cos 2 \theta & 0 \\
\beta \sin 2 \theta & (\alpha-\gamma) \sin 2 \theta \cos 2 \theta & \alpha \sin ^{2} 2 \theta+\gamma \cos ^{2} 2 \theta & 0 \\
0 & 0 & 0 & \gamma
\end{array}\right]
$$

with

$$
\begin{gathered}
\alpha=\frac{p_{x}^{2}+p_{y}^{2}}{2} \\
\beta=\frac{p_{x}^{2}-p_{y}^{2}}{2} \\
\gamma=p_{x} p_{y}
\end{gathered}
$$

where $\theta$ is the linear polarizer's transmission axis orientation, the quantities $p_{x}$ and $p_{y}$ are the absorption coefficients of the orthogonal optical axes $\left(0 \leqslant p_{x}, p_{y} \leqslant\right.$ $1)$. The total intensity of the emerging light $\left(S_{0}^{\prime}, S_{1}^{\prime}, S_{2}^{\prime}, S_{3}^{\prime}\right)$ after passing the linear polarizer is represented by its first Stokes parameter $S_{0}^{\prime}$ :

$$
I=S_{0}^{\prime}=\alpha \cdot S_{0}+\beta \cos 2 \theta \cdot S_{1}+\beta \sin 2 \theta \cdot S_{2}
$$


For a given linear polarizer with reliable physical properties, we can assume its absorption coefficients $\left(p_{x}, p_{y}\right)$ constant. Therefore, mathematically, at least three intensity measurements, through linear polarizers with three different transmission axis orientations, are needed to extract the incident light's partial linear polarization information fully included in the first three Stokes parameters. To determine the optimal number of intensity measurements $N$, a popular strategy is to use a large number of measurements (e.g. $N=16$ ) to reduce the system speckle noise and improve the quality of Stokes images, which are reconstructed with the extracted Stokes parameters for each pixel of the twodimensional photo-sensing array [11]. However, the approach of micropolarizer array, similar to that used in a color filter array, trades-off spatial resolution to allow for the acquisition of the first three Stokes components in a single frame and concretely, each micropolarizer makes only one of the $N$ intensity measurements and $N$ micropolarizers are dedicated to one pixel of the Stokes images to be reconstructed. Although this spatial resolution loss by a factor of $N$ could be alleviated by examining the intensity values of neighboring pixels and adopting interpolation algorithms, it will become extremely difficult even unreliable for a large value of $N$. In addition, in a recently appeared literature [12], Goudail et al. indicate that some noise is independent of even increases with $N$ such as additive noise, and the optimum value of $N$ results from a compromise between the robustness to different noise sources. Here we choose an $N$ value of 4 with the micropolarizers' transmission axis orientations denoted as $\left(\theta_{1}, \theta_{2}, \theta_{3}, \theta_{4}\right)$ and the intensity measurements can be expressed in matrix form:

$$
\left[\begin{array}{l}
I_{1} \\
I_{2} \\
I_{3} \\
I_{4}
\end{array}\right]=\left[\begin{array}{c}
\alpha \beta \cos 2 \theta_{1} \beta \sin 2 \theta_{1} \\
\alpha \beta \cos 2 \theta_{2} \beta \sin 2 \theta_{2} \\
\alpha \beta \cos 2 \theta_{3} \beta \sin 2 \theta_{3} \\
\alpha \beta \cos 2 \theta_{4} \beta \sin 2 \theta_{4}
\end{array}\right] \cdot\left[\begin{array}{l}
S_{0} \\
S_{1} \\
S_{2}
\end{array}\right]
$$

From Eq. (6), we can see that, a judicious choice of $\theta$ for each micropolarizer can significantly reduce the complexity of the Stokes parameters computation circuitry's silicon implementation. Fig. 1 illustrates the adopted micropolarizer array pattern with the micropolarizers' transmission axis orientations along $0^{\circ}$, $90^{\circ}, 45^{\circ}$ and $-45^{\circ}$, respectively, which leads to a simplified extraction of the first three Stokes parameters.

\section{3 "Guest-Host" Micropolarizer Array}

\section{1 "Guest-Host" Interactions in Nematic Liquid Crystals}

Nematic liquid crystals (NLC) consist of rod-like organic molecules, whose regional ordering is characterized by the parallel alignment of molecules, along their long molecular axes. A well-known property of nematic liquid crystals is that the orientation of the molecules can be controlled with an external electric field. Furthermore, Heilmeier discovered that by controlling the molecular orientation of a nematic "host" material, the properties of the "guest" materials mixed 
with the nematic "host" can be controlled [13]. In this chapter, we propose a UVsensitive sulfonic azo-dye SD1 film-based non-contact photoalignment technique to fabricate high-resolution submicron thin "guest-host" micropolarizer arrays. Instead of applying an external electric field to orient the NLC "host" molecules [13], we adopt a non-contact high resolution photoalignment technique based on a UV-sensitive sulfonic-dye-1 (SD1) film, used in liquid crystal displays (LCDs) [14]. As depicted in Fig. 2 (A), a substrate, representing the image sensor, is first

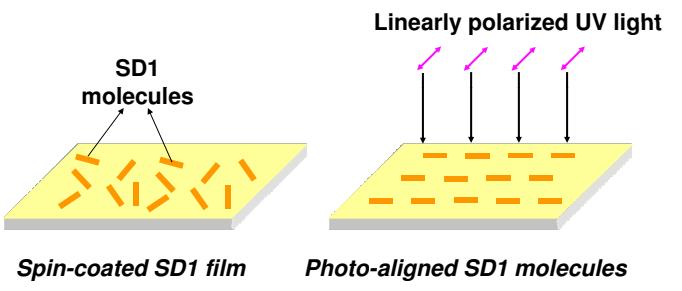

(A)

(B)

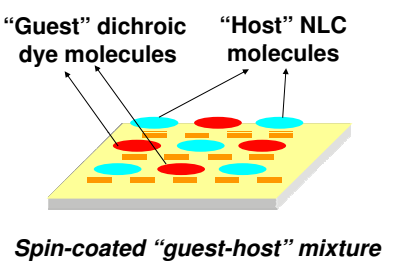

(C)

Fig. 2. Photoalignment of "host" NLC molecules and "guest" dichroic dye molecules.

spin-coated with this SD1 film. After subsequent irradiation by linearly polarized UV light, photoalignment of SD1 molecules occurs [Fig. 2 (B)] with the SD1's long molecular axes perpendicular to the polarization direction of projected linearly polarized UV light. The "guest-host" mixture of dichroic dye and NLC is then spin-coated on top of the SD1 film, which will act as an alignment layer for the NLC "host" molecules and the dichroic dye "guest" molecules. Fig. 2 (C) depicts this reorientation process with "host" and "guest" molecules aligned with SD1 molecules.

\subsection{Fabrication}

To demonstrate the proposed "guest-host" technology, we fabricated the micropolarizer array with the pattern shown in Fig. 1. The latter enables the extraction of full partial linear polarization information [10]. As shown, the "guest-host" micropolarizer array exhibits a $2 \times 2$ pattern comprising $0^{\circ}, 90^{\circ}, 45^{\circ}$ and $-45^{\circ}$ micropolarizers (Fig. 1). To enable the optical characterization of the fabricated micropolarizer array, a transparent glass substrate was used instead of the siliconbased opaque CMOS image sensor substrate. The detailed fabrication steps of the "guest-host" micropolarizer array can be summarized as follows:

1. Organic contaminants were removed from the surface of the transparent glass substrate, using an ultraviolet-ozone (UVO) cleaning machine (Jelight 144AX).

2. An SD1 solution was then spin-coated onto the glass at $800 \mathrm{rpm}$ for $10 \mathrm{~s}$ then $3000 \mathrm{rmp}$ for 40s. In order to eliminate particle impurities, the solution of 
SD1 in dimethylformamide (DMF) with a concentration of $1 \%$ by weight was filtered before the spin-coating.

3. The glass substrate was then baked at $110^{\circ} \mathrm{C}$ for $20 \mathrm{~min}$ to remove the remaining solvent and strengthen the adhesion of the SD1 material to the substrate.

4. The spin-coated SD1 layer on the glass substrate was subsequently photoaligned with the customized "photoalignment master" applied, which is actually a patterned UV-regime metal-wire-grid polarizer from Moxtek Inc. This "photoalignment master", featuring $5 \mu m \times 5 \mu m$ pixel pitch, enables one-step UV-photoalignment of the SD1 layer making the fabrication process simple, cost-effective and high resolution with no misalignment errors. The UV-exposure duration was $15 \mathrm{~min}$ and the UV light intensity at $365 \mathrm{~nm}$ was around $5.6 \mathrm{~mW} / \mathrm{cm}^{2}$. As a result, SD1 molecules were photo-aligned in different microdomains along $0^{\circ}, 90^{\circ}, 45^{\circ}$ and $-45^{\circ}$, respectively.

5. After patterning the NLC photoalignment SD1 layer, a mixture of the dichroic dye solution and the NLC solution (with a mass ratio of 1:1) was spin-coated on top of the patterned SD1 layer at a speed of 800rpm for $5 \mathrm{~s}$ then 3000rpm for $30 \mathrm{~s}$.

6. Next, the substrate with the spin-coated "guest-host" mixture was baked at $50^{\circ} \mathrm{C}$ for $3 \mathrm{~min}$ to eliminate the solvents.

7. Finally, a UV light with an intensity of $2 \mathrm{~mW} / \mathrm{cm}^{2}$ and a wavelength of $254 \mathrm{~nm}$, which is not within SD1's sensitive spectrum and cannot thus reorient SD1 molecules, is applied for 3min to polymerize the NLC "host" material and provide improved protection against changing environmental conditions.

\section{Experimental Results}

In order to examine each micropolarizer domain, the fabricated "guest-host" micropolarizer array sample was back-illuminated by a microscope's white light source. A broadband linear polarizer (from Moxtek Inc.) was inserted and rotated between the white light source and the fabricated sample to provide four different polarized inputs: $0^{\circ}$ linearly polarized, $90^{\circ}$ linearly polarized, $45^{\circ}$ linearly polarized and $-45^{\circ}$ linearly polarized. According to [10], the normalized Stokes parameters $\left(S_{1} / S_{0}, S_{2} / S_{0}\right)$ of the four different polarized inputs are (1, $0),(-1,0),(0,1)$ and $(0,-1)$, respectively. Fig. 3 presents the sample's microphotographs examined by a linear polarization analyzer and recorded by the microscope's camera system. Note that $0^{\circ}, 90^{\circ}, 45^{\circ}$ and $-45^{\circ}$ micropolarizers appear dark as expected when the input is $90^{\circ}, 0^{\circ},-45^{\circ}$ and $45^{\circ}$ linearly polarized, respectively. In addition, a micropolarizer pitch as compact as $5 \mu \mathrm{m} \times 5 \mu \mathrm{m}$ is achieved. The overall "guest-host" micropolarizer array thickness including the SD1 photoalignment layer was measured by a surface profiler (Tencor P10) and found to be $0.95 \mu \mathrm{m}$. Furthermore, the fabricated micropolarizer array was characterized by measuring the four important figures of merit: transmittances $\left(T_{\|}, T_{\perp}\right)$ and absorbances $\left(A_{\|}, A_{\perp}\right)$. Measurements were performed using 


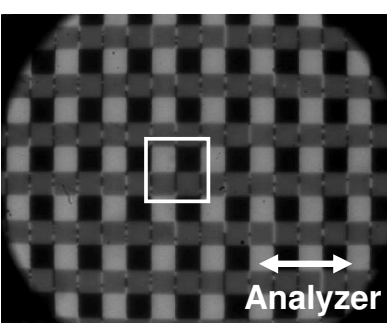

(A)

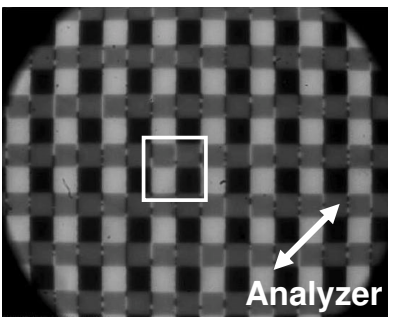

(C)

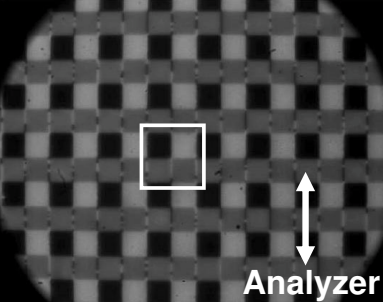

(B)

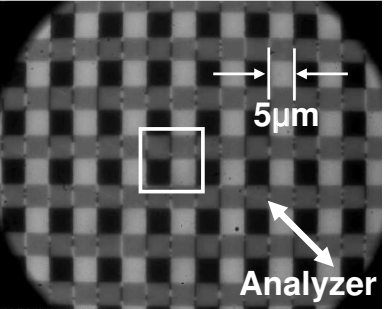

(D)

Fig. 3. Microphotographs of the fabricated "guest-host" micropolarizer array, inspected by a linear polarization analyzer along the following orientations: (A) 0 degree; (B) 90 degree; (C) 45 degree; (D) -45 degree.

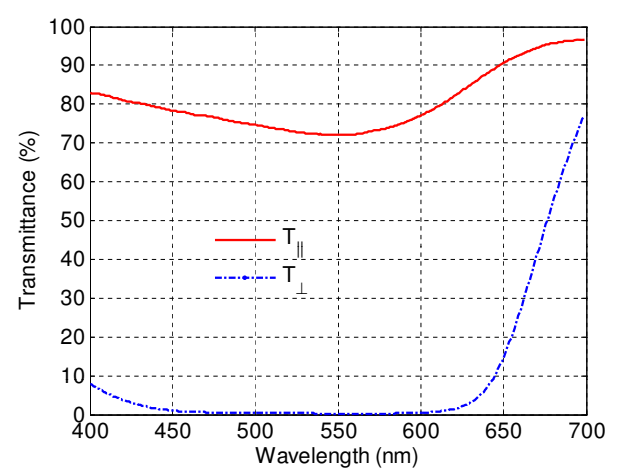

(A)

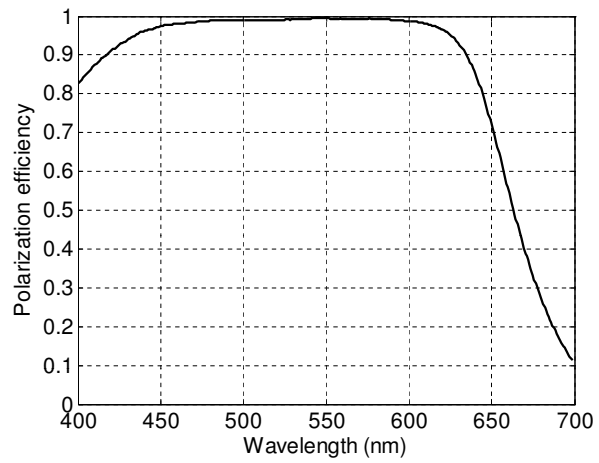

(B)

Fig. 4. Spectral measurement results: (A) the major and minor principal transmittances $T_{\|}, T_{\perp} ;(\mathrm{B})$ polarization efficiency $P E$. 
a polarization state generator (PSG) comprising a mini deuterium halogen light source (DT-Mini-2-GS from Mikropack GmbH) and a broadband linear polarizer (from Moxtek Inc.). This PSG can provide linearly polarized input light with wavelengths ranging from $400 \mathrm{~nm}$ to $700 \mathrm{~nm}$. Since the micropolarizer pitch is in the micrometer scale, which is much smaller than the PSG's laser beam, we fabricated unpatterned "guest-host" linear polarizer samples $(2.5 \mathrm{~cm} \times 2.0 \mathrm{~cm})$ together with the micropolarizer arrays to cover the PSG's laser beam and enable the characterization of the fabricated micropolarizers. Fig. 4 (A) shows the spectral measurement results of both the major and the minor principal transmittances $T_{\|}, T_{\perp}$. The major principal transmittance is seen to range from $71.9 \%$ $(551 \mathrm{~nm})$ to $96.8 \%(699 \mathrm{~nm})$ with an average of $80.3 \%$ across the whole visible spectrum (i.e. from $400 \mathrm{~nm}$ to $700 \mathrm{~nm}$ ). The corresponding average $P E$ across the whole visible spectrum is 0.863 with the maximum $P E$ equal to 0.996 at a wavelength of $545 \mathrm{~nm}$ and the minimum $P E$ equal to 0.114 at a wavelength of $699 \mathrm{~nm}$ [Fig. 4 (B)]. In this chapter, the spectrum band, for which $P E$ exceeds 0.90 , ranges from $417 \mathrm{~nm}$ to $635 \mathrm{~nm}$. This corresponds to $73 \%$ of the whole visible spectrum. This enables high-quality monochromatic or achromatic polarization image sensing applications. Ongoing efforts are focusing on the development and synthesis of dichroic dyes, which are more sensitive to the visible spectrum towards the UV and infrared (IR) ends.

\section{Conclusion}

We have fabricated and characterized a high-resolution "guest-host" micropolarizer array with dichroic dye as the "guest" and polymerizable NLC as the "host". Experimental results demonstrate that micropolarizer arrays exploiting "guest-host" interactions can offer higher resolution $(5 \mu m \times 5 \mu \mathrm{m}$ pixel pitch), submicron thickness but also superior optical performance across the whole visible spectrum, with averaged major principal transmittance and polarization efficiency of $80.3 \%$ and 0.863 , respectively. This is achieved by controlling the "guest" molecular orientation through the photoalignment of "host" molecules. The proposed non-contact micropolarizer array fabrication technology prevents mechanical damage, electronic charge or contamination to the substrate. Furthermore, it is simple and cost-effective, requiring only a single UV-exposure of the spin-coated "guest-host" mixture through a "photoalignment master". It is also compatible with standard CMOS process, enabling the integration of a "guest-host" micropolarizer array over a CMOS image sensor to realize the concept of a low cost single-chip polarization camera.

\section{Acknowledgment}

The authors would like to thank the support from the Research Grant Council of Hong Kong SAR, P. R. China (Ref. GRF610608). 


\section{References}

1. N. A. V. Beare, T. E. Taylor, S. P. Harding, S. Lewallen and M. E. Molyneux, "Malaria retinopathy: a newly established diagnostic sign in severe malaria," $\mathrm{Am}$. J. Trop. Med. Hyg., vol. 75, no. 5, pp. 790-797, 2006.

2. M. C. W. Campbell, C. J. Cookson, J. M. Bueno, A. N. Seaman and M. L. Kisilak, "Confocal Polarimetry Measurements of Tissue Infected with Malaria," Frontiers in Optics, OSA Technical Digest (CD), 2007.

3. A. G. Andreou and Z. K. Kalayjian, "Polarization Imaging: Principles and Integrated Polarimeters," IEEE Sens. J., vol. 2, no. 6, pp. 566-576, 2002.

4. J. Guo and D. Brady, "Fabrication of thin-film micropolarizer arrays for visible imaging polarimetry," Appl. Opt., vol. 39, no. 10, pp. 1486-1492, 2000.

5. V. Gruev, A. Ortu, N. Lazarus, J. Van de Spiegel and N. Engheta, "Fabrication of a Dual-Tier Thin Film Micro Polarization Array," Opt. Express, vol. 15, no. 8, pp. 4994-5007, 2007.

6. M. Momeni and A. H. Titus, "An Analog VLSI Chip Emulating Polarization Vision of Octopus Retina," IEEE Trans. on Neur. Netw., vol. 17, no. 1, pp. 222-232, 2006.

7. C. K. Harnett and H. G. Craighead, "Liquid-crystal micropolarizer array for polarization-difference imaging," Appl. Opt., vol. 41, no. 7, pp. 1291-1296, 2002.

8. V. Gruev, J. Van der Spiegel and N. Engheta, "Nano-wire Dual Layer Polarization Filter," Proc. of ISCAS, pp. 561-564, 2009.

9. X. Zhao, F. Boussaid, A. Bermak and V. G. Chigrinov, "Thin Photo-Patterned Micropolarizer Array for CMOS Image Sensors," IEEE Photon. Technol. Lett., vol. 21, no. 12, pp. 805-807, 2009.

10. D. Goldstein, Polarized Light, 2nd Edition, New York: Marcel Dekker, 2003.

11. I. J. Vaughn and B. G. Hoover, "Noise reduction in a laser polarimeter based on discrete waveplate rotations," Opt. Express, vol. 16, no. 3, pp. 2091-2108, 2008.

12. F. Goudail and A. Beniere, "Estimation precision of the degree of linear polarization and of the angle of polarization in the presence of different sources of noise," Appl. Opt., vol. 49, no. 4, pp. 683-693, 2010.

13. G. H. Heilmeier and L. A. Zanoni, "Guest-Host Interactions in Nematic Liquid Crystals: A New Electro-Optic Effect," Appl. Phys. Lett., vol. 13, no. 3, pp. 91-92, 1968.

14. V. Chigrinov, E. Prudnikova, V. Kozenkov, H. Kwok, H. Akiyama, T. Kawara, H. Takada and H. Takatsu, "Synthesis and properties of azo dye aligning layers for liquid crystal cells," Liq. Cryst., vol. 29, no. 10, pp. 1321-1327, 2002. 